\title{
Prognostic Stage IB Breast Cancer AJCC v8
}

National Cancer Institute

\section{Source}

National Cancer Institute. Prognostic Stage IB Breast Cancer A/CC v8. NCI Thesaurus. Code C139558.

Stage IB includes: (T1, N0, M0, G1, HER2 Status: Negative, ER Status: Positive, PR Status: Negative); (T1, N0, M0, G1, HER2 Status: Negative, ER Status: Negative, PR Status: Positive); (T1, NO, MO, G2, HER2 Status: Positive, ER Status: Positive, PR Status: Negative); (T1, N0, M0, G2, HER2 Status: Positive, ER Status: Negative, PR Status: Any); (T1, NO, M0, G2, HER2 Status: Negative, ER Status: Negative, PR Status: Positive); (T1, N0, M0, G3, HER2 Status: Positive, ER Status: Negative, PR Status: Any); (T1, N0, M0, G3, HER2 Status: Negative, ER Status: Positive, PR Status: Positive); (T 0-1, N1 mi, M0, G1, HER2 Status: Negative, ER Status: Positive, PR Status: Negative); (T0-1, N1 mi, M0, G1, HER2 Status: Negative, ER Status: Negative, PR Status: Positive); (T0-1, N1 mi, M0, G2, HER2 Status: Positive, ER Status: Positive, PR Status: Negative); (T0-1, N1 mi, M0, G2, HER2 Status: Positive, ER Status: Negative, PR Status: Any); (T 0-1, N1 mi, M0, G2, HER2 Status: Negative, ER Status: Negative, PR Status: Positive); (T 0-1, N1 mi, M0, G3, HER2 Status: Positive, ER Status: Negative, PR Status: Any); (T 0-1, N1 mi, M0, G3, HER2 Status: Negative, ER Status: Positive, PR Status: Positive); (T2, N0, MO, G1-3, HER2 Status: Positive, ER Status: Positive, PR Status: Positive); (T2, N0, M0, G1-2, HER2 Status: Negative, ER Status: Positive, PR Status: Positive); (T1, N1, M0, G1-3, HER2 Status: Positive, ER Status: Positive, PR Status: Positive); (T1, N1, M0, G1-2, HER2 Status: Negative, ER Status: Positive, PR Status: Positive); (T2, N1, M0, G1, HER2 Status: Negative, ER Status: Positive, PR Status: Positive); (T2, N1, M0, G2, HER2 Status: Positive, ER Status: Positive, PR Status: Positive); (T 0-2, N2, M0, G1-2, HER2 Status: Positive, ER Status: Positive, PR Status: Positive); (T3, N1-2, M0, G1, HER2 Status: Positive, ER Status: Positive, PR Status: Positive); (T3, N1-2, M0, G2, HER2 Status: Positive, ER Status: Positive, PR Status: Positive). T0: No evidence of primary tumor. T1: Tumor measuring $20 \mathrm{~mm}$ or less in greatest dimension. T2: Tumor measuring more than $20 \mathrm{~mm}$, but not more than $50 \mathrm{~mm}$ in greatest dimension. T3: Tumor measuring more than $50 \mathrm{~mm}$ in greatest dimension. NO: No regional lymph node metastasis is identified or isolated tumor cell clusters (ITCS) are identified only. N1: T umor with 
micrometastases; or metastases in 1-3 axillary lymph nodes; and/or clinically negative internal mammary nodes with micrometastases or macrometastases by sentinel lymph node biopsy. N1 mi: Micrometastases (approximately 200 cells, larger than $0.2 \mathrm{~mm}$, but none larger than $2.0 \mathrm{~mm}$ ). N2: Tumor with metastases in 4-9 axillary lymph nodes; or positive ipsilateral internal mammary lymph nodes by imaging in the absence of axillary lymph node metastases. G1: Low combined histologic grade (favorable); SBR score of 35 points. G2: Intermediate combined histologic grade (moderately favorable); SBR score: 6-7 points. G3: High combined histologic grade (unfavorable); SBR score of 8-9 points. M0: No clinical or radiog raphic evidence of distant metastases. Imaging studies are not required to assign the M0 category. (AJCC 8th ed.) 